\title{
$\mathrm{Tl}_{0.5} \mathrm{~Pb}_{0.5} \mathrm{Sr}_{1.6} \mathrm{Ba}_{0.4} \mathrm{Ca}_{2}\left(\mathrm{Cu}_{1-x} \mathrm{Fe}_{x}\right)_{3} \mathrm{O}_{9+\delta}$ 体系中 的铁元素替代和超导电性 ${ }^{*}$
}

许祝安 张宣嘉 庄飞** 李阳 $* * *$ 焦正宽 张其瑞

(浙江大学物理系, 杭州 310027)

\section{关键词 $\mathrm{Tl}$ 系 1223 相超导体 $\mathrm{Fe}$ 元素替代 载流子浓度}

在 $\mathrm{Tl}$ 系超导铜氧化合物中, 除了合成晶体结构中含双层 $\mathrm{TlO}$ 层的 $\mathrm{Tl}_{2} \mathrm{Ba}_{2} \mathrm{CuO}_{6}(\mathrm{Tl} 2201)$, $\mathrm{Tl}_{2} \mathrm{Ba}_{2} \mathrm{CaCu}_{2} \mathrm{O}_{8}$ ( Tl 2212) 和 $\mathrm{Tl}_{2} \mathrm{Ba}_{2} \mathrm{Ca}_{2} \mathrm{Cu}_{3} \mathrm{O}_{10}$ ( $\mathrm{Tl} 2223$ ) 等高 $T_{\mathrm{c}}$ 相以外, 还合成了只含 T1-O 单层 的超导相, 其分子式分别为 $\mathrm{Tl}(\mathrm{Ba}, \mathrm{Sr})_{2} \mathrm{CuO}_{5}(\mathrm{Tl1} 1201) 、 \mathrm{Tl}(\mathrm{Ba}, \mathrm{Sr})_{2} \mathrm{CaCu}_{2} \mathrm{O}_{7}$ (T11212)和 Tl $(\mathrm{Ba}, \mathrm{Sr})_{2}$ $\mathrm{Ca}_{2} \mathrm{Cu}_{3} \mathrm{O}_{9}(\mathrm{Tl1223})^{[1]}$, 常写成如下通式: $\mathrm{Tl}(\mathrm{Ba}, \mathrm{Sr})_{2} \mathrm{Ca}_{n-1} \mathrm{Cu}_{n} \mathrm{O}_{2 n+3}(n=1,2$ 和 3 等 $)$.

$n \geqslant 3$ 的 $\mathrm{Tl}$ 系超导相中存在两种不等价的 $\mathrm{Cu}$ 晶位. 一般把 $\mathrm{Cu}-\mathrm{O}$ 五配位 (配位多面体为 金字塔形的正四方雉体) 中的 $\mathrm{Cu}$ 晶位称为 $\mathrm{Cu}(2)$ 位, 把 $\mathrm{Cu}-\mathrm{O}$ 四配位 (即中间的 $\mathrm{CuO}_{2}$ 层) 中的 $\mathrm{Cu}$ 晶位称为 $\mathrm{Cu}(1)$ 位.

本文报道以 +3 价的 $\mathrm{Fe}$ 部分地替代 $\mathrm{Tl}$ 系 1223 相中的 $\mathrm{Cu}$ 所引起的正常态输运性质和超 导电性的变化. 发现体系的 Hall 载流子浓度 $n_{\mathrm{H}}$ 和 $T_{\mathrm{c}}$ 均随铁含量增加而下降. 实验结果表 明, 在该替代体系中, $n_{\mathrm{H}}$ 的减小是 $T_{\mathrm{c}}$ 下降的主要原因. 文中还进一步讨论了 $\mathrm{Cu}(2) \mathrm{O}_{2}$ 平面和 $\mathrm{Cu}(1) \mathrm{O}_{2}$ 平面对高 $T_{\mathrm{c}}$ 超导电性的相对重要性.

\section{1 实验方法}

采用固相反应法制备 $\mathrm{Tl}_{0.5} \mathrm{~Pb}_{0.5} \mathrm{Sr}_{1.6} \mathrm{Ba}_{0.4} \mathrm{Ca}_{2}\left(\mathrm{Cu}_{1-x} \mathrm{Fe}_{x}\right)_{3} \mathrm{O}_{9+\delta}(x=0,0.01,0.02,0.03,0.04$ 和 $0.05)$ 样品. 首先将化学纯的 $\mathrm{CaCO}_{3}, \mathrm{SrCO}_{3}, \mathrm{BaCO}_{3}$ 和 $\mathrm{CuO}$ 粉末, 按化学计量比充分混和, 研 磨均匀, 在 $890{ }^{\circ} \mathrm{C}$ 焙烧 $30 \mathrm{~h}$ 以形成 $\mathrm{Sr}_{1.6} \mathrm{Ba}_{0.4} \mathrm{Ca}_{2} \mathrm{Cu}_{3} \mathrm{O}_{y}$ 中间相, 将它作为母相, 再掺人适量 $\mathrm{Tl}_{2} \mathrm{O}_{3}, \mathrm{PbO}$ 和 $\mathrm{Fe}_{2} \mathrm{O}_{3}$, 再次充分研磨均匀, 压片, 于 $940{ }^{\circ} \mathrm{C}$ 通氧气气氛下烧结 $10 \mathrm{~h}$, 然后随炉冷 却至室温.

利用日本理学 $X$ 射线衍射仪 (配 $\mathrm{Cu}$ 靶辐射) 测量样品的室温粉末 $X$ 射线衍射谱. 利用 加速 Mössbauer 谱仪进行 Fe 的室温 Mössbauer 谱测量. 样品的电阻和交流磁化率随温度的 变化曲线分别由标准直流四引线法和互感法测定. Hall 效应测量中使用电磁铁产生的 2.0 $\mathrm{T}$ 的磁场, Hall 电压和温度数据均由 Kethley 181 型纳伏直流电压表读取, 测量过程中样

\footnotetext{
1995-01-23 收稿, 1995-05-20 收修改稿

*国家超导攻关资助项目

** 杭州师范学院物理系, 杭州 310012

*** 北京科学技术大学材料物理系, 北京 100083
} 
品的温漂小于 $\pm 5 \mathrm{mK}$.

\section{2 实验结果和讨论}

所有样品的 X 射线衍射结果表明, 铁含量 $x$ 从 $0 \sim 0.05$ 的各样品均为单相的 1223 相化 合物. 这些结果说明 Fe 确实进人了 T11223 相的晶格中.

从样品的电阻和交流磁化率的温度关系测量得到超导转变临界温度 $T_{\mathrm{c}}$ (这里 $T_{\mathrm{c}}$ 定义为电 阻转变的中点温度, 与交流磁化率转变的起始温度基本一致) 随 $x$ 的变化和图 1 所示. 图中 的短竖线代表了转变宽度. $T_{\mathrm{c}}$ 从 $x=0$ 时的 $122 \mathrm{~K}$ 单调地下降到 $x=0.05$ 时的 $90.5 \mathrm{~K}$.

从 Hall 效应测量 (在 $135 \mathrm{~K}$ 进行) 得出的载流子浓度 $n_{\mathrm{H}}$ 随 $x$ 的变化如图 2 所示. 这里 $n_{\mathrm{H}}=\frac{1}{e R_{H}}$. 显然, $\mathrm{Fe}$ 替代 $\mathrm{Cu}$ 引起了载流子浓度的迅速下降.

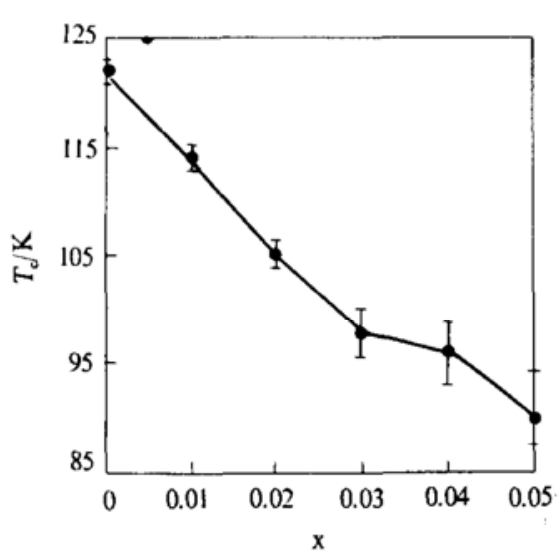

图 $1 \mathrm{Tl}_{0.5} \mathrm{~Pb}_{0.5} \mathrm{Sr}_{1.6} \mathrm{Ba}_{0.4} \mathrm{Ca}_{2}\left(\mathrm{Cu}_{1-x} \mathrm{Fe}_{x}\right)_{3} \mathrm{O}_{9+\delta}$ 体系的 $T_{c}$ 随 $x$ 的变化关系 竖短线为转吩宽度

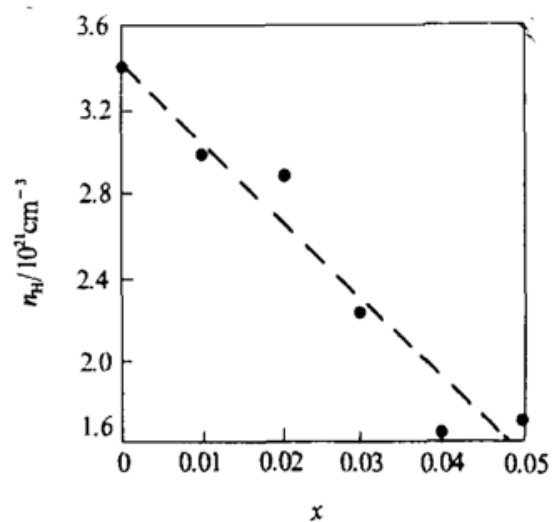

图 $2 \mathrm{Tl}_{0.5} \mathrm{~Pb}_{0.5} \mathrm{Sr}_{1.6} \mathrm{Ba}_{0.4} \mathrm{Ca}_{2}\left(\mathrm{Cu}_{1-x} \mathrm{Fe}_{x}\right)_{3} \mathrm{O}_{9+\delta}$ 体系 的 $n_{\mathrm{H}}$ 随 $x$ 的变化关系

样品的窒温 Mössbauer 谱可用两套亚谱进行拟合, 结果见表 1. 从同质异能移位和四极 䢃裂数值来分析, $\mathrm{Fe}(1)$ 和 $\mathrm{Fe}(2)$ 分别占据了 $\mathrm{Cu}(1)$ 和 $\mathrm{Cu}(2)$ 位, 均为 $\mathrm{Fe}^{3+}$ 态, 且 $\mathrm{Fe}$ 对 $\mathrm{Cu}(1)$ 位 的占据率远大于对 $\mathrm{Cu}(2)$ 位的占据率, 即 $\mathrm{Fe}$ 优先占据 $\mathrm{Cu}$ 的平面晶位 $\mathrm{Cu}(1)$ 位.

表 $1 \mathrm{Tl}_{0.5} \mathrm{~Pb}_{0.5} \mathrm{Sr}_{1.6} \mathrm{Ba}_{0.4} \mathrm{Ca}_{2}\left(\mathrm{Cu}_{1-x} \mathrm{Fe}_{x}\right)_{3} \mathrm{O}_{y}$ 体系的 Mössbauer 谱超精细相互作用参量

\begin{tabular}{c|ccc|ccc}
\hline 替代量 & \multicolumn{3}{|c|}{ 亚谱 I $(\mathrm{Fe}(\mathrm{l}))$} & \multicolumn{3}{c}{ 亚谱 2 (Fe(2)) } \\
\hline \multirow{2}{*}{$x$} & $\begin{array}{c}\text { IS } \\
\left(10^{-3} \mathrm{~m} \cdot \mathrm{s}^{-1}\right)\end{array}$ & $\begin{array}{c}\text { QS } \\
\left(10^{-3} \mathrm{~m} \cdot \mathrm{s}^{1}\right)\end{array}$ & $\begin{array}{c}\text { Relative } \\
\text { Abundanc }\end{array}$ & $\begin{array}{c}\text { IS } \\
\left(10^{3} \mathrm{~m} \cdot \mathrm{s}^{-1}\right)\end{array}$ & $\begin{array}{c}\text { QS } \\
\left(10^{-3} \mathrm{~m} \cdot \mathrm{s}^{\prime}\right)\end{array}$ & $\begin{array}{c}\text { Relative } \\
\text { Abundance }\end{array}$ \\
\hline 0.01 & 0.296 & 1.258 & 78.8 & 0.284 & 0.495 & 21.2 \\
0.02 & 0.230 & 1.283 & 69.6 & 0.199 & 0.535 & 30.4 \\
0.03 & 0.253 & 1.297 & 61.6 & 0.230 & 0.548 & 38.4 \\
\hline
\end{tabular}

$\mathrm{Fe}$ 替代 $\mathrm{Cu}(1)$ 位引起载流子浓度的系统下降, $T_{\mathrm{c}}$ 同时下降, 这与 $\mathrm{YBa}_{2} \mathrm{Cu}_{3} \mathrm{O}_{7-\delta}(\mathrm{Y} 123)$ 中 $\mathrm{Fe}$ 替代 $\mathrm{Cu}-\mathrm{O}$ 链中的 $\mathrm{Cu}(1)$ 位的效应很相似 ${ }^{[2,3]}$. 在 $\mathrm{Y} 123$ 相中, $\mathrm{Fe}$ 替代 $\mathrm{Cu}(1)$, 引起顶角氧原子 $\mathrm{O}(4)$ 的位置变化, 使 $\mathrm{Cu}-\mathrm{O}$ 链中的空穴不能转移到 $\mathrm{CuO}_{2}$ 平面, 导致 $\mathrm{CuO}_{2}$ 平面中可迁移载流 子浓度减小, 因而 $T_{\mathrm{c}}$ 也随之下降. 而 $\mathrm{Zn}$ 择优替代 $\mathrm{Y} 123$ 相中的 $\mathrm{Cu}(2)$ 位, 起拆对效应, 虽然载 
流子浓度变化很小, $T_{\mathrm{c}}$ 也很快下降 ${ }^{[2]}$. 在 $\mathrm{Y} 123$ 相中 $\mathrm{Cu}(2) \mathrm{O}_{2}$ 平面是对高 $T_{\mathrm{c}}$ 超导电性负责的主 要导电平面, 而 $\mathrm{Cu}(1)-\mathrm{O}$ 链则处于次要地位, 相当于 “载流子库” . 所以存在着 $\mathrm{Cu}(2) \mathrm{O}_{2}$ 平面外 和 $\mathrm{Cu}(2) \mathrm{O}_{2}$ 平面内两类不同的元素替代效应机制 ${ }^{[2]}$. 同样地, 在 $\mathrm{T} 11223$ 相中 $\mathrm{Fe}$ 替代 $\mathrm{Cu}(1)$ 位, 导致迁移载流子浓度下降, $T_{\mathrm{c}}$ 同时下降. 这应属于 $\mathrm{Cu}(2) \mathrm{O}_{2}$ 平面外的元素替代效应机制, 通过影响载流子浓度而影响 $T_{\mathrm{c}}$. 所以作者认为, 711223 相中对高 $T_{\mathrm{c}}$ 超导电性负责的主要导 电平面是 $\mathrm{Cu}(2) \mathrm{O}_{2}$ 平面, 而 $\mathrm{Cu}(1) \mathrm{O}_{2}$ 层处于相对次要的地位, 起 “载流子库” 和对两层 $\mathrm{Cu}(2) \mathrm{O}_{2}$ 平面提供耦合的作用.

据 Di Stasio 等人 ${ }^{[4]}$ 对 $\mathrm{Tl}_{2} \mathrm{Ba}_{2} \mathrm{Ca}_{n-1} \mathrm{Cu}_{n} \mathrm{O}_{2 n+4}(n \geqslant 3)$ 体系中空穴浓度在各层中分布的研究, 可迁移空穴主要分布在 $\mathrm{Cu}-\mathrm{O}$ 五配位的 $\mathrm{Cu}(2) \mathrm{O}_{2}$ 平面中, 这两层 $\mathrm{Cu}(2) \mathrm{O}_{2}$ 平面是发生高 $T_{\mathrm{c}}$ 超 导电性的主要导电层. 而中间的一层 $\mathrm{Cu}(1) \mathrm{O}_{2}$ 平面中只含较少的载流子. Trokiner 等人间对 $(\mathrm{Bi}, \mathrm{Pb})_{2} \mathrm{Sr}_{2} \mathrm{Ca}_{2} \mathrm{Cu}_{3} \mathrm{O}_{y}$ 体系的 ${ }^{17} \mathrm{O}$ 的 $\mathrm{NMR}$ 研究也证实中间一层 $\mathrm{Cu}(1) \mathrm{O}_{2}$ 平面的载流子浓度比 $\mathrm{Cu}(2) \mathrm{O}_{2}$ 平面的少 $15 \% \sim 25 \%$. T11223 相中 $\left[\mathrm{Cu}(2) \mathrm{O}_{2}-\mathrm{Ca}-\mathrm{Cu}(1) \mathrm{O}_{2}-\mathrm{Ca}-\mathrm{Cu}(2) \mathrm{O}_{2}\right]$ 的结构与 $\mathrm{T} 12223$ 相和 Bi2223 相中相应的单元十分类似. 故可以认为, 在 T11223, 2223 和 $\mathrm{Bi} 2223$ 相中, $\mathrm{Cu}(2) \mathrm{O}_{2}$ 平面是主要的导电层, 是发生高 $T_{\mathrm{c}}$ 超导电性的平面. 而 $\mathrm{Cu}(1) \mathrm{O}_{2}$ 平面则处于较次要的地位. +3 价 $\mathrm{Fe}$ 替代 $\mathrm{Cu}(1)$ 的效应也证实了上述观点.

综上所述, $\left(\mathrm{Tl}_{0.5} \mathrm{~Pb}_{0.5}\right) \mathrm{Sr}_{1.6} \mathrm{Ba}_{0.4} \mathrm{Ca}_{2}\left(\mathrm{Cu}_{1-x} \mathrm{Fe}_{x}\right)_{3} \mathrm{O}_{9+\delta}$ 体系的迁移载流子浓度 $n_{\mathrm{H}}$ 和超导临界温 度 $T_{\mathrm{c}}$ 随 $\mathrm{Fe}$ 含量 $x$ 增加而同时下降. $n_{\mathrm{H}}$ 减小是 $T_{\mathrm{c}}$ 下降的主要原因. $\mathrm{Cu}(2) \mathrm{O}_{2}$ 平面是产生高 $T_{\mathrm{c}}$ 超导电性的主要导电层. $\mathrm{Cu}(1) \mathrm{O}_{2}$ 层中的载流子浓度较小, $\mathrm{Cu}(1) \mathrm{O}_{2}$ 面处于较次要的地位, 起调 节 $\mathrm{Cu}(2) \mathrm{O}_{2}$ 面中载流子浓度和为两层 $\mathrm{Cu}(2) \mathrm{O}_{2}$ 面之间提供耦合的作用.

\section{参考 文 献}

1 Parkin S S P, Lee V Y, Nazzal A I et al. $\mathrm{Tl}_{1} \mathrm{Ca}_{n-1} \mathrm{Ba}_{2} \mathrm{Cu}_{n} \mathrm{O}_{2 n+3}(n=1,2,3)$ : a new class of crystal structures exhibiting volume superconductivity at up to $\approx 110 \mathrm{~K}$. Phys Rev Lett, 1988, 61(6):750 753

2 Zhang Q R, Xu Z A, Wang J S et al. Elemental substitution effects in high- $T_{\mathrm{c}}$ superoonductor $\mathrm{YBa}_{2} \mathrm{Cu}_{3} \mathrm{O}_{7-\delta}$. Science in China, Series A, 1993, 36(11): $1376 \sim 1385$

3 Xu Z A, Ouyang S, Wang J S et al. Superconductivity of $\mathrm{Y}_{1-x} \mathrm{Ca}_{x} \mathrm{Ba}_{2} \mathrm{Cu}_{3-x} \mathrm{Fe}_{x} \mathrm{O}_{7-\delta}$. Chinese Science Bulletin, 1992, $37(18): 1520 \sim 1523$

4 Di Stasio M, Muller K A, Pietronero L. Nonhomogeneous charge distribution in layered high- $T_{\mathrm{c}}$ superconductors. Phys Rev Lett, 1990, 64(23): $2827 \sim 2830$

5 Trokiner A, LeNoc L, Schneck J. ${ }^{17} \mathrm{O}$ nuclear-magnetic-resonance evidenœe for distinct carrier densities in the two types of $\mathrm{CuO}_{2}$ planes of $(\mathrm{Bi}, \mathrm{Pb})_{2} \mathrm{Sr}_{2} \mathrm{Ca}_{2} \mathrm{Cu}_{3} \mathrm{O}_{y}$. Phys Rev B, 1991, 44(5):2 426 2429 